•综述・

\title{
植物-微生物互惠共生：演化机制与生态功能
}

\author{
卢明镇* \\ (Santa Fe Institute, Santa Fe NM 87501, USA)
}

\begin{abstract}
摘要: 植物一微生物互惠共生是一种特殊的合作形式, 在整个生命和陆地生态系统的演化历史中起着至关重要的 作用。在全球环境变化背景下, 植物和微生物间的互惠共生对生态系统功能的维持具有重要意义。尽管合作/互惠 共生如此重要, 在生物学中却存在着对它的历史偏见与忽视。特别地, 尽管互惠共生的理论与建模发展已有较长 的历史，但不同学科分支间仍存在着多种不同的观点。本综述从两个看似对立的视角概述植物一微生物互惠共生的 概念框架, 即微生物学家关心的微观机制和生态系统生态学家关注的宏观影响。宏观模型通常从一组过于简单的 假设出发, 便于理论分析。但微观机制是开展定量预测的基础, 因此新一代基于过程的宏观模型需嵌入微观机制, 这对预测全球变化下的生态系统响应至关重要。此外, 希望本文也可以吸引更多学者关注合作/互惠的重要作用, 并将这一概念应用于解决其他生态学和社会学问题。
\end{abstract}

关键词: 植物-微生物互惠共生; 理论生态学; 模型; 合作; 博亦论; 生物市场理论

\section{Plant-microbe mutualism: Evolutionary mechanisms and ecological functions}

\author{
Mingzhen Lu* \\ Santa Fe Institute, Santa Fe NM 87501, USA
}

\begin{abstract}
Plant-microbe mutualism, a special form of cooperation, has been crucial throughout the evolutionary history of life and terrestrial ecosystems. With human activities changing the condition of Earth's surface at an unprecedented rate and scale, we expect this ancient bond between plants and microbes to continue to play a key role. Yet, despite its importance, there has been a historical bias towards cooperation/mutualism in biology, and a general underrepresentation in mathematical biology/theoretical ecology. Moreover, even though theoretical representation of mutualism has come a long way, there exists multiple disparate perspectives with diverse associated scientific communities, among which interaction has been limited. This review focuses on two seemingly opposite schools of perspectives: microbiologists' perspective that zooms in for the microscale mechanisms vs. ecosystem ecologists' perspective that zooms out for the macroscale consequences. Macroscale models often start with a simple set of naive assumptions. But over time microscale mechanisms (once understood well) will eventually be incorporated into newer-generation process-based large models, greatly enhancing our ability to quantitatively predict our future. I hope this review can facilitate this process, a process that will only become more important against the backdrop of rapid global change. Lastly, but perhaps more broadly, I hope this review will attract more attention to the important role of cooperation/mutualism, a concept that we can maybe leverage to solve a range of other broader problems in ecology and our society.
\end{abstract}

Key words: Plant-microbe mutualism; theoretical ecology; model; cooperation; game theory; biological market theory

互惠共生不仅是地球上所有生命形式的重要 组成部分, 而且是陆地生态系统早期演化的基本驱
动力。早在植物登陆之前, 地衣(真菌和藻类共生) 就已经在陆地表面繁衍, 并大大加快了岩石风化形

收稿日期: 2020-10-25; 接受日期: 2020-12-02

* 通讯作者 Author for correspondence. E-mail: mingzhen.lu@santafe.edu. ORCID: https://orcid.org/0000-0002-8707-8745 
成土壤的速度。正如现代生态系统中地衣能够促进 植物在原生演替中生长一样, 这种菌藻共生关系为 地球早期植物的登陆和繁荣奠定了基础。

早期植物开始在陆地上生长时 ( 4.5 亿年前), 面临着如何从土壤基质中吸收水分以及可溶性养 分的挑战。植物通过与原始的从枝菌根真菌形成互 惠关系来克服这一困难, 即通过真菌菌丝从土壤中 获取水分和养分(Strullu-Derrien et al, 2014)。真菌菌 丝平均直径约为 $5 \mu \mathrm{m}$, 而现代植物的细根在全球尺 度上平均约为 $300 \mu \mathrm{m}$ (Ma et al, 2018)。这意味着在 生物量相同的情况下, 这些植物共生者的搜索长度 可达到植物根的3,600倍。由于共生菌根对植物资源 供应的重要作用, 植物和真菌之间的这种战略合作 对陆地系统早期森林的出现至关重要。

早期森林的繁荣给陆生植物带来了另一个挑 战: 由于难以分解的木质素和纤维素的堆积, 越来
越多的营养物质被锁在有机碎屑中。从二叠纪开始, 某些腐生菌类群通过分解有机质并释放锁定养分 解决了这个问题(Floudas et al, 2012)。松科植物的早 期物种与这些腐生菌( 1.4-1.8亿年前)的后代形成 了战略互惠关系，逐渐演变成了现代外生菌根植 物。这种独特的互惠关系进一步增强了植物获取养 分的能力, 并促进植物向新的生态位扩张, 如温带 森林(Martin et al, 2016)。

现代热带雨林在大约 6,500万年前出现，由于 较高的生产力, 它们对土壤养分有较高的需求, 这 对热带雨林植物构成了新的独特的挑战。维管植物, 特别是豆科植物, 开始与能够固定大气氮的菌株形 成稳定的互惠关系(Werner et al, 2015)。固氮植物的 出现使植物能够将新的氮带入系统, 使生态系统免 受氮的限制。

\section{植物－微生物互惠共生关系虽然在古生物学文}
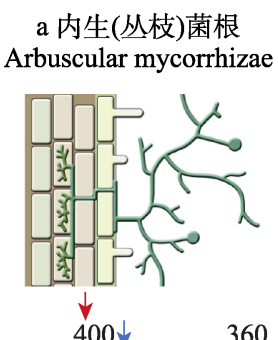

b 早期森林

Early forest

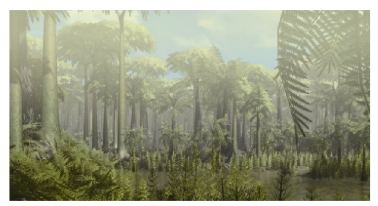

300 c 外生菌根 Ectomycorrhizae

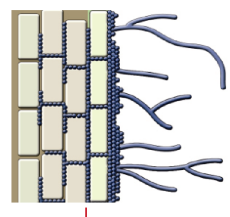

$\mathrm{d}$ 有花植物 Flowering plants e 固氮根瘤 Nitrogen fixing nodule

泥盆纪 石炭纪叠纪
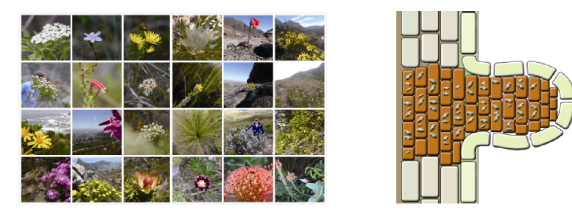

$145 \downarrow$

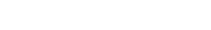

65

(百万年)

白严纪

Cretaceous

图 1 陆地生态系统中植物-微生物互惠共生的演化。a. 在登陆后不久, 陆地植物与内生菌根真菌(AMF)的祖先形成了共生 关系(Strullu-Derrien et al, 2014)。图中展示了单个根尖(左，浅绿色的表皮根细胞和浅棕色的皮层细胞)和 AMF 菌丝是如何形 成细胞内结构的。b. 早期森林出现于木本维管植物占优势的早泥盆纪(Willis \& McElwain, 2014)。c. 陆地植物与外生菌根真 菌(EMF) (Cairney, 2000; Martin et al, 2016)建立稳定共生关系。EMF 是能够分解木质素的腐生真菌的后代，帮助植物获取 有机质中被锁住的养分。d. 受环境条件变化的选择，早白严纪出现了有花植物，是植物适应性创新的一个里程碑(Willis \& McElwain, 2014)。e. 植物创新的另一个里程碑事件发生在地下, 白严纪-古近纪界线不久之后(Werner et al, 2015), 植物与固 氮菌形成了互惠共生关系。这些细菌(居住在橙色的细胞中)可以打开氮气的三键, 为植物供应可利用的氮。共生关系 $\mathrm{a} 、 \mathrm{c} 、$ e 用红色箭头指向地质时间，地质史关键事件 b、d 则使用蓝色箭头。该图修改自 Lu 和 Hedin (2019)的图 1。a、c、e 图作者 为孙渏南, $\mathrm{b}$ 图来自布朗大学的 Andrew Lesile, $d$ 图来自作者本人。

Fig. 1 Evolution of plant-microbe mutualism in terrestrial ecosystems. a. Land plants formed associations with early ancestors of arbuscular mycorrhizal fungi (AMF) soon after the plant's colonization of terrestrial ecosystems (Strullu-Derrien et al, 2014). The schematic illustrates how an individual root tip (left, showing epidermal root cells in light green and cortical cells in light brown) and AMF mycelial forms intracellular structure. AMF hyphae is magnitude thinner than even the thinnest plant roots, allowing them superb ability to access soil resources from the porous soil matrix. b. The early forest emerged in the early Devonian after woody vascular plants gained dominance (Willis \& McElwain, 2014). c. Land plants formed associations with ectomycorrhizal fungi (EMF) (Cairney, 2000), the descendant of wood-decaying fungi, aiding plants in accessing nutrients that otherwise would be locked into organic matter. d. Selected by the changing environmental condition, flowering plants emerged during the early Cretaceous as a milestone for plants' adaptive innovation (Willis \& McElwain, 2014). e. Another milestone for plant innovation happened belowground, shortly after the Cretaceous-Paleogene boundary Werner et al (2015), with plants forming mutualistic associations with nitrogen-fixing bacteria. These bacteria (housed in these orange cells) can breakdown the triple bond of $\mathrm{N}_{2}$ gas and supply plants with plant available forms of nitrogen. The geological timings of mutualistic relationships $\mathbf{a}, \mathbf{c}, \mathbf{e}$ are indicated by red arrows, while that of geological events $\mathbf{b}, \mathbf{d}$ by blue arrows. This figure is modified based on Figure 1 in Lu \& Hedin (2019). Illustration in a, c, e, from Yinan Sun, $\mathbf{b}$ from Andrew Lesile of Brown University, and $\mathbf{d}$ from the author. 
献中常常被忽视, 但综上可以看出, 它在地球历史 上许多重要的里程碑事件里都起到了重要的推动 作用。通过帮助植物克服资源的限制, 植物和微生 物的互惠共生关系促进了植物生命向新的、更具挑 战性的生态位扩展, 最终促成了我们目前观察到的 植物、动物和栖息地的丰富多样性。

\section{生物学和理论生态学领域的历史偏见}

尽管互惠共生关系很重要, 但与竞争相比得到 的关注很少。不仅实验性研究中如此, 在理论生态 学和数学生物学领域更为突出 (Goh, 1979; May, 1974)。考虑到理解和利用互惠共生关系的重要性, 我们有必要先弄清楚这种历史偏见是如何形成的。

最明显的原因是达尔文进化论产生的文化惯 性。达尔文这位生物学中最有影响力的人物将竞争 的观点置于生物学中最有影响力的理论的中心位 置(Darwin, 1859)。毫不夸张地说，达尔文确立了竞 争的主导地位, 认为竞争是生物学和生态学中最重 要的驱动力。达尔文在他的《物种起源》中确实也 注意到了互惠共生的重要性: “一朵花和一只蜜蜂 可能会慢慢地，同时地或相继地，进化并以最完美 的方式相互适应。而这种适应是基于个体产生对彼 此有利的结构变异, 而使它们更好地生存”。这是他 在整本书中对互惠关系最深的也是唯一的一次阐 述。随后发展起来的与进化相关的理论(Wilson, 1975; Wilson \& Sober, 1994)则更强调合作，但从未 获得与《物种起源》类似的影响力。

从更深层的角度看, 人们倾向于关注竞争而忽 视互惠共生的原因植根于二者辩证组合的本质, 即 组内合作、组间竞争。人们倾向认可团体之间的竞 争, 因为它更明显, 往往更吸引人的关注。然而, 推 动每一次竞争升级的隐藏力量却是集团内的合作, 但对局外者来说这并不容易观察到。这种情况在人 类行为的进化中经常出现(Bowles \& Gintis, 2011), 在植物-微生物系统中更是如此(例如, 观察两棵树 的竞争比观察帮助它们竞争的共生体要容易得多)。

而在理论生态学中, 人们对互惠共生缺乏关注 还有另一层原因, 即与Lotka-Volterra方程(以下称 为 $\mathrm{L}-\mathrm{V}$ 方程) 的数学特性有关。对于理论生态学家来 说, L-V 方程对理解种群和群落生态学具有重要意 义。我们最为熟知的 $L-V$ 方程是用于描述捕食者-猎
物动力学和种间资源竞争的。然而，从L-V方程被提 出的那一刻起, 它其实还可以描述合作和互惠共生 (Lotka, 1925)。那么为什么L-V方程很少应用于植物 一微生物互惠共生或合作的领域呢? 我们在这里使 用L-V方程的一般形式(Lotka，1925的现代版)作一 说明:

$$
\begin{aligned}
& \frac{d N_{1}}{d t}=N_{1}\left(r_{1}+a_{11} N_{1}+a_{12} N_{2}\right) \\
& \frac{d N_{2}}{d t}=N_{2}\left(r_{2}+a_{21} N_{1}+a_{22} N_{2}\right)
\end{aligned}
$$

其中, $a_{i i}$ 表示每个物种对自身的影响, $a_{i j}$ 表示物种 $\mathrm{j}$ 对i的影响。一般而言, $a_{i i}$ 由于密度依赖性而呈负值。 因此, 两个物种相互作用的动态是由 $a_{i j}$ 的符号决 定。具体来说，我们可以把两种物种的相互作用分 为以下几类: 竞争 $\left(a_{12}, a_{21}<0\right)$ 、互利共生 $\left(a_{12}, a_{21}>\right.$ $0)$ 、捕食或寄生 $\left(a_{12}<0, a_{21}>0\right)$ 。May (1974)在其关 于生态系统稳定性和复杂性的经典著作中指出, 互 惠共生在理论生态学领域被忽视是因为历史原因, 而非生物学原因。更具体地说，他认为早期建立的 Lotka-Volterra系统难以维持互惠关系的稳定性。比 较图 2 中的a图和 $\mathrm{b}$ 图可见, 参数的唯一区别是 $\mathrm{b}$ 图 (无限种群)比a图有更强的互惠交互项。换句话说, 较强的互惠作用可引起种群的无限增长, 因此互惠 关系成为了 “导致群落动态不稳定的因素” (May, 1974)。

此外，植物的多个个体和多个共生体菌株之间 的生物相互作用在自然界中几乎是普遍的(Moeller \& Neubert, 2016), 而L-V方程最初被提出是来处理 两两相互作用。虽然L-V框架可以很容易扩展到包 含多个物种的相互作用(Goh，1979; Neuhauser \& Fargione, 2004), 但这会使它失去最初让它受欢迎 的简洁性。

综上, 我们可以理解为什么在生物学文献中特 别是在理论生态学(其中种群生物学是核心)中, 涉 及到互惠关系的篇幅仍然相对不足。然而, 关于植 物-微生物互惠的研究在两种不同视角下得到了迅 速发展: 微生物学家聚焦于植物-微生物相互作用 的机制, 而生态系统生态学家聚焦于植物-微生物 相互作用的宏观影响。本文将概述这两种视角及其 相关的数学工具(表1)。 

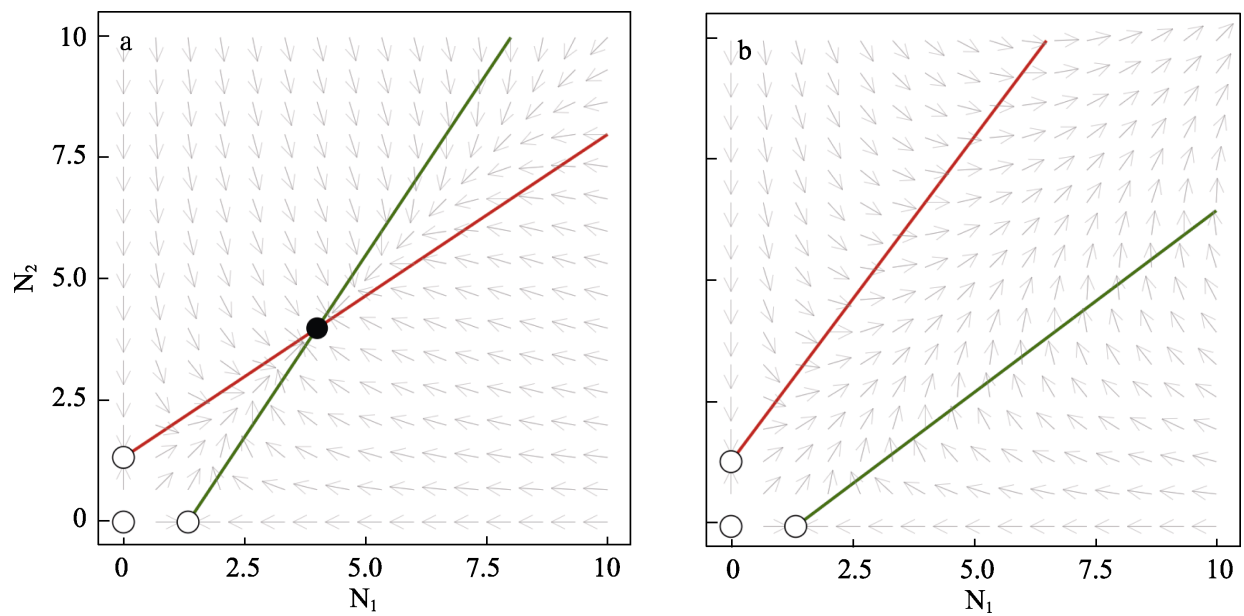

图2 互惠种群 $N_{1}$ 和 $N_{2}$ 的相平面。a. 当雅各比行列式 $\operatorname{det}(J)>0$ 时, 相互作用导致稳定的非平凡平衡 $\left(r_{1}=r_{2}=2, a_{11}=a_{22}=-1.5\right.$, $\left.a_{12}=a_{21}=1\right)$ 。b. 当雅各比行列式 $\operatorname{det}(\mathrm{J})<0$ 时, 相互作用导致种群数量无限增长的非稳定动态 $\left(r_{1}=r_{2}=2, a_{11}=a_{22}=-1.5\right.$, $a_{12}=a_{21}=2$ )。种群 $N_{1}$ 的零增长等值线用绿色, $N_{2}$ 的零增长等值线用红色。黑色填充圆表示稳定平衡点, 白色填充圆表示非稳 定和半稳定(鞍点)平衡点, 灰色箭头表示种群变化方向。绘图软件Julia 1.4.1。

Fig. 2 Phase planes of mutualistic interactions between population $\mathrm{N}_{1}$ and $\mathrm{N}_{2}$. a. The mutualistic interaction leads to stable nontrivial equilibrium when $\operatorname{det}(J)>0\left(r_{1}=r_{2}=2, a_{11}=a_{22}=-1.5, a_{12}=a_{21}=1\right)$. b. The mutualistic interaction leads to non-stable dynamics (infinite populations size) when $\operatorname{det}(\mathrm{J})<0\left(\mathrm{r}_{1}=\mathrm{r}_{2}=2, \quad \mathrm{a}_{11}=\mathrm{a}_{22}=-1.5, \mathrm{a}_{12}=\mathrm{a}_{21}=2\right)$. The nullcline of population $\mathrm{N}_{1}$ is labeled in green and $\mathrm{N}_{2}$ in red. Black-filled circle denotes stable equilibrium while white-filled circles denote non-stable and half-stable (saddle points) equilibria. Plots are made in Julia 1.4.1.

表1 本综述中涉及的建模方法

Table 1 A comparison of modeling approaches covered in this review

\begin{tabular}{llll}
\hline 研究视角 & 建模方法 & 优点 & 弱点 \\
Perspective & Modeling approach & Strength & Weakness \\
\hline 种群生物学 & L-V方程 & 熟悉, 简洁 & 互惠导致种群不稳定性 \\
Population biology & Lotka-Volterra equations & Familiarity and simplicity & Infinite population due to mutualism \\
微生物生物学 & 迭代囚徒困境 & 简单, 通用性 & 缺乏种群动态, 缺乏伙伴选择, 对称设置 \\
Microbial biology & Iterated Prisoner's Dilemma & Simplicity and generality & Lack of population dynamics, lack of partner \\
& & & choice, and symmetric setup \\
& 生物市场理论 & 非对称设置, 伙伴选择 & 各种数学工具的混合 \\
& Biological market theory & Asymmetry and partner choice & Lack of simplicity, mixture of tools \\
生态系统生态学 & 现象学 & 计算效率高 & 原理机制不足 \\
Ecosystem ecology & Phenomenology & Computational efficiency & Not mechanistic \\
& 优化 & 概念简单 & 任意选择的目标函数 \\
& Optimization & Conceptual simplicity & Arbitrary goal function \\
& 自适应动态 & 可以模拟生物对变化的响应 & 计算成本高, 难扩展到大尺度模型中
\end{tabular}

\section{2 微生物学家的观点: 关注微观机制}

接下来要介绍的这个视角是一个多元化的研 究领域，关注的是植物和微生物相互作用的机理。 具体来说, 来自这一视角的研究者更多地关注植物 一微生物相互作用是如何出现(emergence)和维持的, 而较少关注这种相互作用对种群动态和生态系统 过程的影响。以下简要介绍这一学派的建模方法, 主要关注博弯论方法(囚徒困境)和生物市场理论。

\section{1 囚徒困境}

自达尔文时代起，互惠共生作用一直是一个令
博物学家和科学家们困扰的难题。根据定义, 互惠 共生包括相互作用的双方从它们的互惠互利者中 获益(b), 同时因为提供服务而产生自身成本(c) (基 于定义, $b>c>0$ )。进化生物学家会很自然地问一个 简单的问题: 如果一个个体“决定”从互惠互利者那 里获利却不付出成本去提供服务(成本 $=0$ ), 将有 何后果? 这种欺骗策略将享受更高的回报, 即 $(b-0)>(b-c)$, 并将随着时间的推移入侵互惠的种 群。既然如此, 那为什么进化史保留了互惠共生呢? 我们可以用一个简单的理想化的二人博弯(表 
2)来表述这一悖论。在这个博弯中, 玩家可以选择 两种策略: 合作和欺骗。这种特殊的设置在博弯论 的文献中被称为“捐赠博亦” (Hilbe et al, 2013), 它 是更为普遍的“目徒困境” (Prisoner’s Dilemma, 简 称 PD)博弯的一种特殊情况。自从贝徒困境首次被 引入科学文献, 它已经成为理解合作的一般动态的 一个理想工具。在 20 世纪 70 年代, 它被引入生物 学, 用来理解利他行为和互惠共生(Trivers, 1971)。

表2 囚徒困境博变特殊情况下的收益矩阵。在每一轮游戏 中, 每个玩家都可以选择合作或欺骗。合作的收益为 $\mathbf{b}$, 成本 为 $\mathbf{c}$, 如果双方都合作, 双方都得到 $\mathbf{b}-\mathbf{c}$ 的回报。如果双方都 不合作, 回报是零。如果一方作弊, 另一方合作, 作弊者没 有支付成本 $\mathbf{c}$ 就得到了效益 $\mathbf{b}$, 合作者支付成本 $\mathbf{c}$ 却没有获得 效益b，博峦的纳什均衡用粗体表示。

Table 2 Payoff matrix for a special case of Prisoner's dilemma game. Each player can choose to cooperate or cheat during each round of this game. The benefit from cooperation is $\mathbf{b}$ and cost of cooperation is $\mathbf{c}$. If both cooperate, each get b-c. If neither cooperate, the payoff is zero. If one cheat while the other cooperate, the cheater get the benefit $\mathbf{b}$ without paying the cost $\mathbf{c}$, and the cooperator pay the cost $\mathbf{c}$ without gaining the benefit $\mathbf{b}$. The Nash equilibrium of this game is bolded.

\begin{tabular}{|c|c|c|}
\hline & $\begin{array}{l}\text { 玩家B (合作) } \\
\text { Player B (Cooperate) }\end{array}$ & $\begin{array}{l}\text { 玩家B (欺骗) } \\
\text { Player B (Cheat) }\end{array}$ \\
\hline $\begin{array}{l}\text { 玩家A (合作) } \\
\text { Player A (Cooperate) }\end{array}$ & $\mathrm{b}-\mathrm{c} ; \mathrm{b}-\mathrm{c}$ & - c; b \\
\hline $\begin{array}{l}\text { 玩家A (欺骗) } \\
\text { Player A (Cheat) }\end{array}$ & $\mathrm{b} ;-\mathrm{c}$ & $0 ; 0$ \\
\hline
\end{tabular}

基于我们对徒困境的理解, 上面提到的悖论 具有一个纳什均衡(Nash equilibrium), 即 $(0,0)$ 。在 这个均衡中, 任何一方都不能通过单方面改变策略 来提高自己的适应度(fitness)。这个均衡告诉我们, 两个玩家都会欺骗, 并且只要满足 $b>c>0$, 欺骗 就是一种进化稳定策略。很明显, 这个均衡并不是 整个系统的最佳解决方案, 因为当把两个玩家的收 益相加时, 2(b-c) $>(b-c)>0$ 。从这个简单的模型中 得出的定性结论显然与我们在自然界中观察到的 情况不一致, 因为植物和微生物之间的互惠共生关 系是数百万年来植物进化的基础, 并且在不同的群 体中都有进化(图1)。这就提出了一个基本问题: 合 作/互惠关系如何在囚徒困境框架内维持?

在囚徒困境框架中实现持续合作的一种方法 是在游戏中进行多轮博弯, 每个玩家都能够“记住” 上一轮所使用的策略并相应地改变自己的策略(迭 代囚徒困境，Iterated Prisoner's Dilemma，简称 IPD)。IPD是理解植物-微生物共生与合作的基础,
被广泛应用。下面我简要介绍IPD数学方法的要点。 表2中收益矩阵的特殊情况可以推广如下：可以定 义 $P^{k}$ 为给定个体 $k(k=\mathrm{A}, \mathrm{B})$ 的回报, 为收益 $\mathrm{b}$ 与成本 $\mathrm{c}$ 的差。收益 $\mathrm{b}$ 是对方 $j$ 投资 $I^{j}$ 的函数 $(k \neq j)$, 成本 $\mathrm{c}$ 是自 身 $(k)$ 投资给对方 $I^{k}$ 的函数:

$$
P^{k}=b\left(I^{j}\right)-c\left(I^{k}\right)
$$

在植物-微生物互惠系统中, $\mathrm{A}$ 可以代表植物宿 主, $\mathrm{B}$ 代表与之共生的微生物, $I^{A}$ 代表植物对共生生 物的碳投资, $I^{B}$ 代表共生体对寄主植物的养分供给。 收益矩阵是由双方的投资组合 $\left(I^{k}, I^{j}\right)$ 唯一决定的。 IPD框架的核心是将时间维度添加到收益方程中:

$$
P_{t}^{k}=b\left(I_{t}^{j}\right)-c\left(I_{t}^{k}\right)
$$

其中 $t$ 代表 $\mathrm{A}$ 和 $\mathrm{B}$ 之间进行的某一轮游戏(图3a)。下一 轮每个玩家的投资 $I_{t+1}^{k}$ 是前一轮游戏的函数:

$$
I_{t+1}^{k}=f\left(I_{t}^{k}, I_{t}^{j}, P_{t}^{k}\right)
$$

函数 $f$ 定义了玩家所采取的迭代策略, 例如著 名的IPD策略“针锋相对” (tit-for-tat)可以表示为 $I_{t+1}^{k}=I_{t}^{j}$ (一般初始投资 $I_{0}$ 会选择合作)。Dobeli (1998)从植物-微生物互惠共生的角度提出了以下 策略:

$$
I_{t+1}^{k}=I_{t}^{k}+b^{k} P_{t}^{k}
$$

其中, 植物根据其先前的回报将其前一轮投资用回 报率 $\mathrm{b}$ 来修正。如果 $\mathrm{b}$ 为正, 则意味着每一个互惠者 (寄主植物和微生物共生体)只要之前的回报为正 (来自交互作用的净收益), 就会增加投资。这是惩罚 欺骗/寄生，鼓励互惠，从而维持合作的重要机制。

综上，博弯论方法尽管构造非常简单，却可以 让我们获得强大的洞察力。然而博弯论方法尤其是 应用最广泛的IPD方法, 存在一些关键的局限性:

首先，自然界中的植物和微生物之间的碳-营 养交换(投资组合) $\left(I^{k}, I^{j}\right)$ 是连续的, 形成一个从寄 生到互惠的连续梯度, 但博弯论方法的默认设置是 针对每个玩家的二元策略，比如开或关。尽管后来 的工作(从20世纪 90 年代后期开始)将二元策略扩展 至连续策略, 但这种扩展常常不可避免地导致模型 变得非常复杂，而简单性恰恰是博弯论方法的优势 所在。

其次，在囚徒困境的框架下，持续的互惠共生 需要同一参与者之间的重复互动。在植物一微生物 互惠共生中, 这意味着植物和微生物通过垂直传 
a

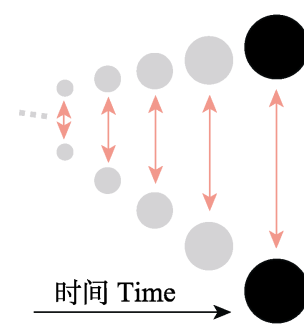

迭代风徒困境

lterated Prisoner's Dilemma $\mathrm{b}$

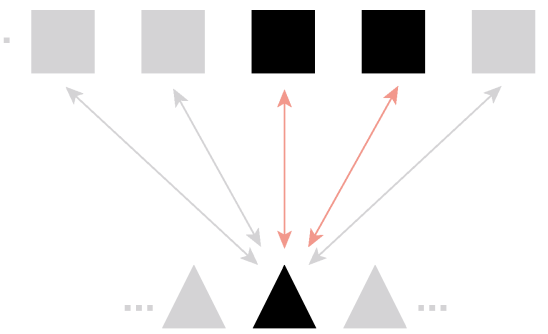

生物市场理论

Biological Market Theory

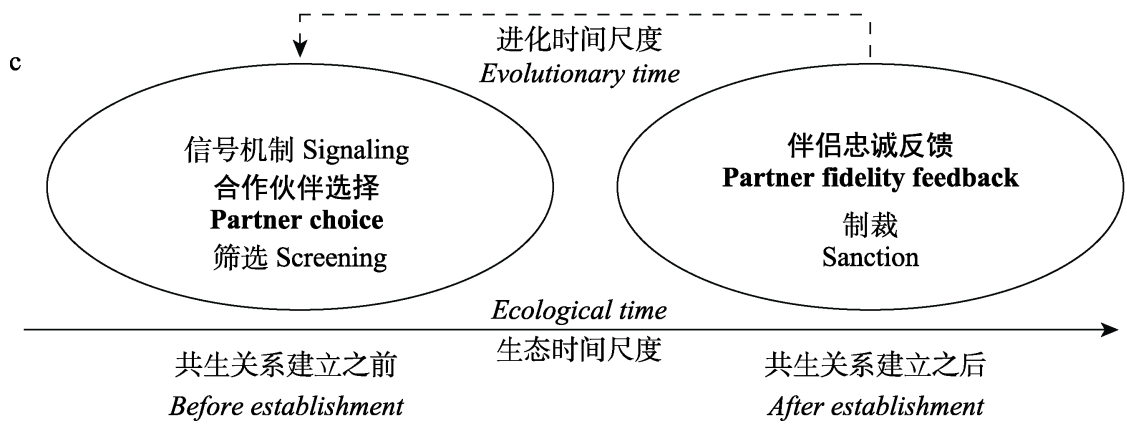

图3 迭代囚徒困境(IDP) (a)与生物市场理论(BMT) (b)的图形说明以及BMT的核心概念(c)。a. 两个玩家随着时间的推移以 对称的方式重复互动, 每个玩家都能够记住最近一次的互动。玩家被标记为相同的填充点, 强调了这种方法固有的对称性(例 如, 蝙蝠之间的种内合作)。b. 两组玩家通过类似于二分网络的形式进行交互作用, 其中一组中的每个玩家都可以与来自另 一组的多个玩家进行交互(为了简化视觉, 这里只显示了部分交互)。这两个组在这里用不同的符号表示(正方形和三角形), 以 强调这种方法内在的不对称性, 这使得它在处理特定种间的互惠互动(例如, 植物与微生物, 蜜蜂与花)时很有优势。BMT模 型的最小设置用浅红色标记, 一共三个玩家, 其中一个玩家与另外两个玩家交互。c. 生物市场理论里面的最重要基本概念可 以从生态时间尺度分成两类(实线时间轴): 合作伙伴选择和伴侣忠诚反馈。这两类概念所对应的生物学过程在进化时间尺度 上是相连的(虚线)。

Fig. 3 Graphical illustration of Iterated Prisoner’s Dilemma (IDP) (a) vs. Biological Market Theory (BMT) (b), and core concepts used in BMT (c). a. Two players interact in a symmetric manner repeatedly over time, with each player being able to remember interactions from the immediate last time step. The players are denoted with the same filled dots, emphasizing the symmetry inherent to this approach (for example, intraspecific cooperation between individual bats). b. Two classes of players interact through a bipartite-graph alike interaction, where each player in one class can interact with multiple players from the other class (for visual simplicity, only part of the interactions are shown here). The two classes are denoted with different symbols here to emphasize the inherent asymmetry of this approach, which makes it unique in dealing with inter-specific mutualistic interaction (for example, plants vs. microbes, bees vs. flowers). The minimal setup of a BMT model is labeled in light red, where 1 player interacts with 2 other. c. The most fundamental concepts in BMT can be divided into two broad classes based on timescale (solid time arrow): partner choice vs. partner fidelity feedback. The biological processes represented by these two classes of concepts are linked over evolutionary time (dashed line).

代，以相同的基因型相互作用(后代树继承的微生 物与母株相同)。但一般认为, 植物-微生物的互利 共生更多地依赖水平传播, 很少通过垂直传播(Noë \& Kiers, 2018)。

最后, 可能也是最重要的一点, 囚徒困境(以及 其他游戏) 的默认设置假设了交互类之间的对称关 系。这种设置适用于解决种内合作问题, 但没有考 虑到种间互惠共生固有的不对称性。例如, 豆科植 物与根瘤菌的相互作用涉及一个寄主与多种菌株 的相互作用(Simms \& Taylor, 2002), 而植物与菌根 的相互作用涉及多个寄主和共生体。

\section{2 生物市场理论}

由于囚徒困境模型的局限性, Noë在研究不对 称求偶系统时提出了生物市场理论(Biological Market Theory, BMT) (Noë, 1990; Noë \& Hammerstein, 1994)。该理论与IPD的关键区别是: 玩家可以根据服务质量的不同在多个玩家之间进 行选择(因此BMT中玩家的最小数量为 3 , 如图3所 示)。目前, BMT已经成为了解植物-微生物互惠共 生及其对环境条件依赖性的最流行的工具。BMT的 核心思想是, 互惠交换在本质上类似于人类社会中 进行产品和服务交易的市场。BMT不是一种具体形 
式的数学方法, 而更多是一种基于一系列假设的概 念框架。Ronald Noë和他的同事在他们的开创性论 文中概述了这些假设(Noë \& Hammerstein，1994), 并在最近的一篇更具有生物学基础的综述中对这 些假设做了更新(Noë \& Kiers, 2018)。在具体的数学 模型上, BMT可以呈现为非常不同的形式, 比如双 人博弯、Tilman的资源竞争框架(de Mazancourt \& Schwartz, 2010)等。

BMT的核心包括两个概念：合作伙伴选择和 伴侣忠实反馈(图3c)。合作伙伴选择指的是在寄主一 共生体关系建立前每一方都可以在多个报价中进 行选择, 评估预期回报并在最有利的回报中做出选 择。但宿主或共生体如何在不执行合作关系之前就 计算预期收益? 这就需要双方通过一些信号来预 测它们未来的收益, 比如使用化学混合物在根瘤菌 和植物宿主之间传递信号。但这些预先建立的机制 存在一个问题, 即作弊者可以侵入系统。伙伴忠实 反馈是确保互惠关系稳定维持的一个重要机制, 即 在合作关系开始执行后, 彼此确保对对方负责。其 要点可以通过公式 6 来理解, 其中 $t+1$ 时刻的相互投 资取决于前一个时间步长的收益。最简单的情况是 一个具有正反馈系数的线性响应(参见等式7)。例如, 如果根瘤菌表现良好, 宿主会通过优先的碳分配奖 励它们(Bever, 2015); 而如果细菌作弊, 则宿主会 通过给予较少的资源来惩罚共生体，通常称为“制 裁” (sanction)。

然而伴侣忠实反馈机制会产生试错成本。理想 情况下, 伴侣会在不需要参与的情况下就预先甄别 有可能作弊的一方。可以想象, 在进化过程中双方 可以从它们建立合作后的表现中学习, 并将合作过 程中的回报与某些信号建立相关函数, 从而进化出 更加微妙的伴侣选择机制。随着时间的推移, 欺骗 行为和对抗措施会不停出现, 就像一场进化的舞 蹈。我们原本预期很强的伴侣选择机制会导致一个 唯一赢家的出现以及随之而来的生物多样性的消 失, 但正是这种进化上的“军备竞赛”维持了互惠共 生关系在物种和群落层面的多样性。相比结构更简 单的博亦论模型, 生物市场理论已经逐渐成为了学 术界用来研究多样性如何维持以及多样性如何受 到环境因素影响的有力工具。

\section{3 未来的发展方向}

任何学科的进步都需要两条腿走路: 经验认识
的发展，以及能够刻画经验认识的理论创新。以下 三个具体方向可能会为微生物学家视角提供助益:

首先，需要更好地理解植物-微生物相互作用 的微尺度生理过程，包括信号通路(Foo et al, 2014)、 资源交换机制(Wipf et al, 2019)、相互作用网络性 (Noë \& Kiers, 2018)等。研究技术的发展无疑会推动 对这些过程的理解。但如果能促进菌根真菌和固氮 领域科研人员之间的交流合作，将显著加快积累经 验认识的速度。

其次，人们越来越多地意识到，“贝徒困境博 亦”框架在很多情形下可能并不是理解合作的最佳 理论框架(Doebeli \& Hauert, 2005)。例如, “雪堆博 亦” 是一种更加微妙的游戏, 基于该游戏无需多重 迭代就可以维持合作。但目前将该模型框架应用于 植物与微生物相互作用的研究还很少。

最后, 需要更强大的数学方法来整合不同类型 模型的优点，同时避免其固有的局限性。尽管还处 于起步阶段, 但网络理论似乎是一个很有前途的方 向，因为它具有描述共生关系的天然复杂性的内在 能力。菌根的相互作用网络和豆科植物-根瘤菌的 相互作用网络都可以被理想化为类似于二分网络, 而这些交互网络所涉及的物种多样性(宿主层面和 共生菌层面)是传统的数学方法无法描述的。在很长 一段时间内，理论生态学在处理生物多样性时的做 法一直是把多样性分解为少量的功能属性, 然后再 用能够描述这些功能属性的简单数学工具来分析 (比如把成百上千种真菌统一看作一个共生菌类群, 然后在博弯模型中用一个玩家来描绘)。而网络模型 的兴起让我们可以憧憬, 在不久的将来我们的理论 工具可以直面物种多样性带来的复杂性。鉴于网络 理论和工具的快速发展(Santos \& Pacheco，2005), 我们有理由相信在植物-微生物互惠共生的研究中 发展网络分析有很好的前景(Montesinos-Navarro et al, 2012; Noe \& Kiers, 2018)。

\section{3 生态系统生态学家的视角: 关注大尺度白}

与微生物学家的视角不同, 生态系统生态学 家、生物地球化学和地球系统建模学者的关注点集 中在群落、生态系统乃至整个生物圈的涌现特性 上。历史上，这些宏观尺度植物一微生物互惠共生的 作用在很大程度上被忽视了。然而, 近来越来越多 
的证据表明植物一微生物的相互作用在塑造宏观尺 度生态系统属性上具有关键作用(Averill et al, 2014; Terrer et al, 2016; Craig et al, 2018; Tedersoo et al, 2020), 因而植物－微生物互惠共生在宏观尺度上的 定量分析正逐渐引起关注。

这些定量分析的核心是碳-营养元素交换的概 念构架, 该构架将对共生体的碳投资视为成本, 将 从共生体中获得的营养素视为收益。Vitousek和 Field (1999)的工作可能是最早从生态系统角度模 拟植物一微生物相互作用的尝试, 其中氮吸收途径 成本被概念化并建立为数学模型。尽管它比较原始 简单, 并且只关注了植物和根瘤菌的相互作用, 但 它从生态系统生态学家和生物地球化学家的角度 抓住了碳一营养交换的核心概念。该方案可以描述 为以下一般形式:

$$
g=f\left(x_{i}, \theta_{j}\right)
$$

其中 $g$ 代表单个植物或整个植被的生长率(通常以净 初级生产量的形式), $x_{\mathrm{i}}$ 是影响增长率的因素, $\theta$ 作 为内在成本，它表征了不同营养吸收途径 $j$ 之间的 成本差异, 如固氮和菌根营养吸收的成本不同。

尽管这一模型提供了刻画碳-营养元素交换过 程的一般性理论构架, 但长期以来研究植物-菌根 相互作用与植物-根瘤菌相互作用的生态学家一直 缺乏交流。这种隔阂从美国生态学会年度会议上两 个领域会议日程的重叠中就可以明显看出。我们必 须把所有地下植物－微生物的互惠共生看作是一系 列进化上相互关联的生物创新(图1), 并将其反映在 一个整合的数学公式中。而一个整合的观点可以让 我们的精力集中在碳和营养物质的通量交换上(从 以植物为中心的角度), 并有利于我们探索植物-微 生物互惠共生对生态系统属性的影响。下文将简要 介绍植物一微生物共生的理论/计算方法, 以及在植 被模式和生物地球化学循环中的应用。

\section{1 植被模式}

植被模式是植物在群落和生态系统尺度上所 呈现出的空间和时间的规律性。很多研究表明, 植 物和微生物之间的相互作用可促进群落水平植被 模式的出现(Jiang et al, 2017; Bennett et al, 2017)。比 如, 植物-真菌互惠共生影响群落构建(community assembly) (Taylor et al, 2019)、生态系统原生和次生 演替早期阶段的群落更迭(Vitousek et al, 2002;
Zheng et al, 2020), 以及在原始热带雨林持续被干 扰和丢失养分的情况下雨林功能的维持(Batterman et al, 2013)。在更大尺度上, 植物-微生物共生在全 球生态学研究中亦有重要作用。经验证据表明, 植 物-微生物共生可影响全球植被的生物地理学模式, 比如植被分布的纬度格局与不同菌根类型的协同 变化(Read, 1991; Read \& Perez-Moreno, 2003)以及 固氮植物在热带地区极高的丰富度(Jenny 1950; Hedin et al, 2009; Vitousek et al, 2002; Gentry, 1988)。然而, 尽管以往研究表明植物-微生物共生 在理解生态系统大尺度时空格局中具重要意义, 但 相关的数学建模却远远落后。一个关键限制是, 在 生态系统尺度上仅仅考虑营养元素和碳的通量交 换是不够的, 必须同时考虑植物-土壤反馈(养分循 环)。

为什么整合植物-微生物互惠以及植物-土壤反 馈如此重要呢? 因为互惠共生关系本身会改变整 个生态系统的营养状况(例如固氮情况下, 向系统 中添加新的氮), 然后通过植物一土壤反馈传递到群 落的动态, 因此最终的植被格局将取决于植物-微 生物互惠共生与养分循环的相互作用。关于植物土壤反馈的理论和概念的文献很早已出现 (DeAngelis, 1992, Wilson \& Agnew, 1992), 但对植 物-土壤反馈和植物-微生物互惠共生的定量整合 则要晚得多(Menge et al, 2009)。Menge等(2009)以生 物地球化学领域的经典箱型模型(DeAngelis, 1992) 为基础, 探讨了不同时间尺度上植物一根瘤菌互惠 共生对生态系统营养限制的影响。在此基础上, 最 新进展不仅整合了植物和根瘤菌的相互作用, 而且 整合了在共生细菌和菌根真菌辅助下的整个植物 营养策略(Lu \& Hedin, 2019)。通过整合植物-土壤 反馈和一系列营养互惠共生作用, 新模型能够解释 和预测自然界中双稳态植被 (见下文解释) 的出现 (Torti et al, 2001; Peh et al, 2011)。

从枝菌根真菌(AMF)型植物主导的森林群落和 外生菌根真菌(EMF)型植物主导的森林群落有时会 同时出现在一个景观地带中, 但它们占据不同的斑 块而不相互混合(图4a, b), 这导致AMF或EMF型植 物丰度呈双峰分布(图4c, d)。Lu和Hedin (2019)提出 的理论框架通过植物一土壤反馈和植物-微生物互 惠共生的相互作用对这一看似奇怪的现象给出了 直观解释。一方面, 菌根共生体的生理机制(图1)表 
明, AMF植物更依赖无机养分, 而EMF植物则依靠 有机养分。另一方面, 最新研究对AMF植物和EMF 植物如何影响植物-土壤养分反馈有了经验认识 (Lin et al, 2017): EMF植物抑制矿化过程, 从而有利 于有机养分的形成, 而AMF植物促进无机养分的产 生。当这两个关键组成部分连接在一起时, 由于植 物与菌根之间的正反馈作用, 出现了一种路径依赖 的现象，或者群落生态学中的“优先效应” (Schoener, 1976; Fukami \& Nakajima, 2011)。具体地说, 当某种 类型的植物群落形成后, 将进一步促进同一植物和 菌根的建植, 最终产生对初始条件敏感的两种稳定 状态(图4e)。

\section{2 生物地球化学循环和全球模拟}

生态系统的营养限制一直是生态系统生态学 和生物地球化学研究的中心议题(Vitousek, 2004)。 在全球变化的背景下, 这一议题变得越来越重要。
最值得注意的是, 随着大气 $\mathrm{CO}_{2}$ 浓度的增加, 植物 对养分的需求不断上升，而越来越多的养分被锁定 在有机物(活着的和死去的)中，陆地植被可能正在 耗尽养分。这种渐进性养分限制的想法最初是针对 氮提出的(Luo et al, 2004), 后来也扩展到其他主要 土壤养分，如磷(Ellsworth et al, 2017)。

如上所述，从宏观角度来看，基于碳和营养素 交易构架的数学模型具有相同的成本效益结构核 心。除了这个共享的核心之外，还可以通过一系列 不同的具体算法来模拟植物的实际生长过程，从简 单算法到复杂算法构成一个梯度(表1)。

在梯度的一端是一种现象学模型，它描述了基 于观察数据的生物学过程, 并常常为特定的生态系 统制定特殊的参数。例如, MySCaN (Orwin et al, 2011)、ANAFORE (Deckmyn et al，2011)和 MYCOFON (Meyer et al, 2010)都可以有效地模拟 a
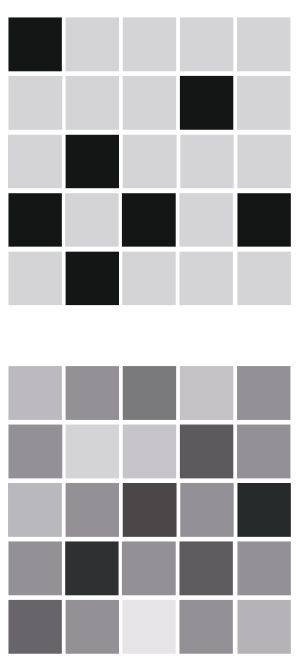
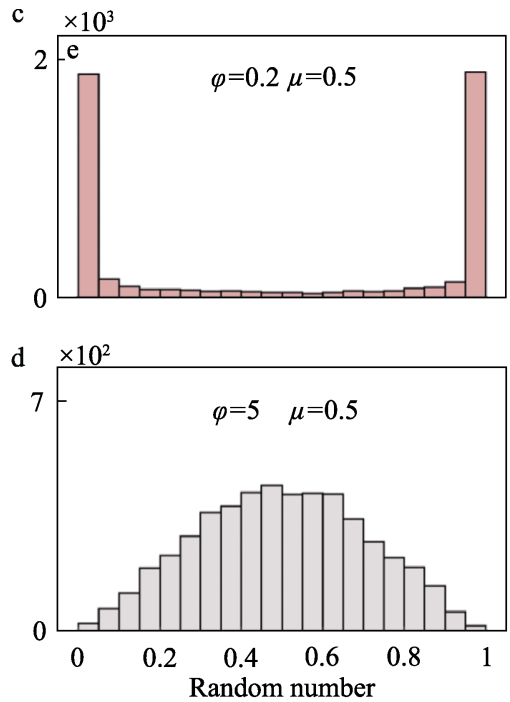

随机数

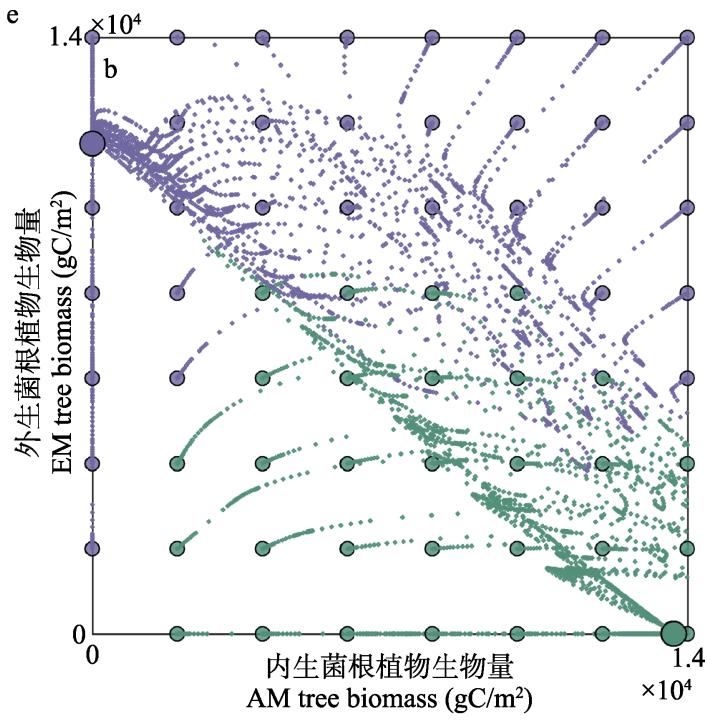

图4 双稳态植被格局及植物-微生物互惠共生的作用。a、b 为植被斑块的景观图, 其中互惠共生类型 $\mathrm{A}$ 和B (在这种情况下, 丛 枝菌根与外生菌根植物)的丰富度表示为色块的灰度。 $\mathrm{a}$ 所呈现的景观中, 双稳态植被状态是存在的, 一块植被要么是 $\mathrm{A}$ 主导 要么是B主导, 而b则是两种互惠共生类型的随机混合。C、d互惠共生关系的分布可以用从beta分布中提取的 5,000 个随机数(代 表5,000个景观斑块)的频率分布来说明: $f(y: \mu, p h i)=c y^{\mu \varphi-1}(1-y)^{(1-\mu) \varphi-1}$ (y表示A的百分比, $\mu$ 作为集中趋势, $\boldsymbol{\phi}$ 为离散系数)。 c图所示的双峰大体可以描绘a图色块的频率分布，而 $\mathbf{d}$ 图的单峰分布可以描绘 $\mathbf{b}$ 图色块的频率分布。 $\mathrm{e}$ 图表示 $\mathrm{A}$ 和B的初始成分 (小点)随着时间的推移而分化成两种不同的稳定状态(大点, 紫色表示EMF, 绿色表示AMF)。图c、d、e摘自Lu和Hedin (2019)。 Fig. 4 Bistable vegetation patterns and the role of plantmicrobe mutualism . a, b. An illustration of a landscape with patches of vegetation, where the abundance of mutualistic interaction A and B (in this case, Arbuscular mycorrhizal vs. ectomycorrhizal symbioses) is denoted by the darkness of the gray hue. a presents a landscape where bistable vegetation states is found where you either find a patch of vegetation extremely high or extremely low in one type of mutualism, whereas $\mathbf{b}$ has a mixture of both mutualistic type in each patch. c, d. The distribution of mutualist abundance can be illustrated using the frequency distribution of 5,000 random numbers (representing 5,000 landscape patches) drawn from a beta distribution: $f(y: \mu, p h i)=c y^{\mu \varphi-1}(1-y)^{(1-\mu) \varphi-1}$ (y indicates percentage of $\mathrm{A}, \mu$ as the central tendency, and $\phi$ as the dispersion coefficient). e. Patches that have different founding composition (small dots) of A and B will over time diverge into two alternative stable states (larger dot, EMF indicated in purple and AMF indicated in green). Panels c, d, e are reproduced from figures published in Lu \& Hedin (2019). 
植物-菌根关系对大尺度生态系统过程的作用。这 种方法的优点是, 只要提供最新的数据其计算效率 就非常高。但是, 这些模型非常依赖于特定的生态 系统, 其中的功能关系是从特定的生态系统总结并 派生出来的，而并不能普遍适用于其他系统。

在梯度的中间, 研究人员假设植物像人类一样 是理性的(借用了经济学的概念), 暗含植物“有意识 地” 优化某些指标的假设。这种优化方法理论上讲 可以应用于更广泛的系统。例如, 可以假设植物从 碳成本最低的途径吸收养分Fisher等(2010), 类似的 技术可追溯到Vitousek和Field (1999)。这个算法在 操作上是简单直接的, 但是我们从经验上知道, 植 物本身有一个比这个简单假设更微妙的“算法”。尽 管有大量更容易获取的可溶性土壤养分, 但固氮植 物在施肥后仍从固氮根瘤获取氮 (代价昂贵) (Menge et al, 2015)。这条研究路线后来的发展借鉴 了物理学中的电阻概念, 将每条养分吸收通道的成 本视为电阻, 将通过每条通道的营养物通量视为电 流(Brzostek et al, 2014; Sulman et al, 2019)。植物的 多种营养获取通道(根系本身、菌根、根瘤等)可以 被视为并联的电路, 将欧姆定律应用于并联电路, 使得仿真更加简单，在某种程度上也更加真实。

优化方法理论的局限性是目标函数选择具有 任意性, 比如碳的增长量、植物的高度或者生物 量。随着环境条件的急剧变化, 最适应者的标准可 能会发生很大的变化。最高的、最重的或者碳含量 最高的可能并不是存活得最好的: 说到底最适应环 境变化的才是最能够存活下来的(Darwin, 1859)。因 此, 在梯度最复杂的一端是自适应动力学方法 (adaptive dynamics), 它能够描述植物适应变化环境 的能力(Diekmann, 2003; Dercole \& Rinaldi, 2008)。 由于分析和计算的复杂性, 自适应动力学方法最近 才被应用到植物－微生物互惠共生的领域，且大多 数工作都局限于相对简单的理论模型。例如, Menge 等(2008)分析了生态系统中固氮策略的进化局限 性。最近, Lu和Hedin (2019) 考虑了植物-菌根和植 物-根瘤菌的互惠共生, 并利用自适应性动力学方 法, 仅仅基于非常简单的外部驱动就能够预测生境 尺度上的植被模式和碳、氮、磷的循环。不过由于 代码复杂性和计算能力的限制, 将这些理论模型扩 展为更具有预测性的全球尺度的过程模型仍比较 困难。

\section{3 未来的发展方向}

从前面介绍的模型的复杂性梯度来看, 越是贴 近现实的算法越局限于简单的小型理论模型，而大 型地球系统模型仍然使用非常具有操作性的极为 简单的算法。更进一步来说，地球系统模型使用的 功能型(functional type)是固定的, 根据收集的数据 预先计算每个特定生态系统的参数。虽然这种方法 从计算速度的角度来看是非常优越的, 但是也带来 了巨大的挑战。由于算法的刚性，大型系统模型的 预测能力被限制在用来训练模型的数据范围内。

地球系统模型的进一步发展会逐渐引入更复 杂更真实的算法，这些算法通过更小的理论模型的 探索变得更成熟。近年来, 新的地球系统模型包含 了各种更加复杂的优化算法(Brzostek et al, 2014; Franklin et al, 2014; Sulman et al, 2019), 以及越来 越现实的生物过程(Fisher et al, 2015; Fisher \& Koven, 2020; Koven et al, 2020)。尽管自适应动力学 方法固有的算法复杂，我们仍可以期待它将成为地 球系统建模工具箱中的主要工具之一。事实上，目 前已发展了基于自适应动力学的地球系统模型，只 不过模拟的是地表过程(Weng et al，2017，2019; Franklin et al, 2020)。笔者乐观地认为, 植物-微生物 互惠共生将很快被纳入采用适应性动力学方法的 大规模过程模型中。

\section{总结}

植物－微生物的互惠共生关系在不同时空尺度 上对塑造生态系统中的关键过程起着至关重要的 作用。然而, 从历史上看, 生物学和生态学长期忽 视了这种控制机制而更关注竞争和寄生。希望本文 能吸引读者更多关注互惠共生的重要性，认识到共 生与竞争是一个动态的相互依存的矛盾体，应该作 为一个整体来研究。因为这个矛盾体的两个组成部 分(就像一个硬币的两面)一起推动了陆地生态系统 的进化和现代生物圈的涌现。

\section{参考文献}

Averill C, Turner BL, Finzi AC (2014) Mycorrhiza-mediated competition between plants and decomposers drives soil carbon storage. Nature, 505, 543-545.

Batterman SA, Hedin LO, van Breugel M, Ransijn J, Craven DJ, Hall JS (2013) Key role of symbiotic dinitrogen fixation in tropical forest secondary succession. Nature, 502, 
224-227.

Bennett JA, Maherali H, Reinhart KO, Lekberg Y, Hart MM, Klironomos J (2017) Plant-soil feedbacks and mycorrhizal type influence temperate forest population dynamics. Science, 355, 181-184.

Bever JD (2015) Preferential allocation, physio-evolutionary feedbacks, and the stability and environmental patterns of mutualism between plants and their root symbionts. New Phytologist, 205, 1503-1514.

Bowles S, Gintis H (2011) A Cooperative Species-Human Reciprocity and Its Evolution. Princeton University Press, Princeton.

Brzostek ER, Fisher JB, Phillips RP (2014) Modeling the carbon cost of plant nitrogen acquisition: Mycorrhizal trade-offs and multipath resistance uptake improve predictions of retranslocation. Journal of Geophysical Research: Biogeosciences, 119, 1684-1697.

Cairney JWG (2000) Evolution of mycorrhiza systems. Naturwissenschaften, 87, 467-475.

Craig ME, Turner BL, Liang C, Clay K, Johnson DJ, Phillips RP (2018) Tree mycorrhizal type predicts within-site variability in the storage and distribution of soil organic matter. Global Change Biology, 24, 3317-3330.

Darwin C (1859) On the Origin of Species. J. Murray, London. de Mazancourt C, Schwartz MW (2010) A resource ratio theory of cooperation. Ecology Letters, 13, 349-359.

DeAngelis DL (1992) Dynamics of Nutrient Cycling and Food Webs. Springer, Dordrecht, the Netherlands.

Deckmyn G, Campioli M, Muys B, Kraigher H (2011) Simulating $\mathrm{C}$ cycles in forest soils: Including the active role of micro-organisms in the ANAFORE forest model. Ecological Modelling, 222, 1972-1985.

Dercole F, Rinaldi S (2008) Analysis of Evolutionary Processes: The Adaptive Dynamics Approach and Its Applications. Princeton University Press, Princeton.

Diekmann O (2003) A beginner's guide to adaptive dynamics. Banach Center Publications, 63, 1, 47-86.

Doebeli M, Hauert C (2005) Models of cooperation based on the Prisoner's Dilemma and the Snowdrift game. Ecology Letters, 8, 748-766.

Ellsworth DS, Anderson IC, Crous KY, Cooke J, Drake JE, Gherlenda AN, Gimeno TE, MacDonald CA, Medlyn BE, Powell JR, Tjoelker MG, Reich PB (2017) Elevated $\mathrm{CO}_{2}$ does not increase eucalypt forest productivity on a low-phosphorus soil. Nature Climate Change, 7, 279-282.

Fisher RA, Muszala S, Verteinstein M, Lawrence P, Xu C, McDowell NG, Knox RG, Koven C, Holm J, Rogers BM, Spessa A, Lawrence D, Bonan G (2015) Taking off the training wheels: The properties of a dynamic vegetation model without climate envelopes, CLM4.5(ED). Geoscientific Model Development, 8, 3593-3619.

Fisher RA, Koven CD (2020) Perspectives on the future of land surface models and the challenges of representing complex terrestrial systems. Journal of Advances in Modeling Earth Systems, 12, doi:10.1029/2018ms001453.

Floudas D, Binder M, Riley R, Barry K, Blanchette RA, Henrissat B, Martínez AT, Otillar R, Spatafora JW, Yadav JS, Aerts A, Benoit I, Boyd A, Carlson A, Copeland A, Coutinho PM, de Vries RP, Ferreira P, Findley K, Foster B, Gaskell J, Glotzer D, Górecki P, Heitman J, Hesse C, Hori C, Igarashi K, Jurgens JA, Kallen N, Kersten P, Kohler A, Kües U, Kumar TKA, Kuo AL, LaButti K, Larrondo LF, Lindquist E, Ling A, Lombard V, Lucas S, Lundell T, Martin R, McLaughlin DJ, Morgenstern I, Morin E, Murat C, Nagy LG, Nolan M, Ohm RA, Patyshakuliyeva A, Rokas A, Ruiz-Dueñas FJ, Sabat G, Salamov A, Samejima M, Schmutz J, Slot JC, St John F, Stenlid J, Sun H, Sun S, Syed K, Tsang A, Wiebenga A, Young D, Pisabarro A, Eastwood DC, Martin F, Cullen D, Grigoriev IV, Hibbett DS (2012) The Paleozoic origin of enzymatic lignin decomposition reconstructed from 31 fungal genomes. Science, 336, 1715-1719.

Foo E, Ferguson BJ, Reid JB (2014) Common and divergent roles of plant hormones in nodulation and arbuscular mycorrhizal symbioses. Plant Signaling \& Behavior, 9, e29593.

Franklin O, Harrison SP, Dewar R, Farrior CE, Brännström A, Dieckmann U, Pietsch S, Falster D, Cramer W, Loreau M, Wang H, Mäkelä A, Rebel KT, Meron E, Schymanski SJ, Rovenskaya E, Stocker BD, Zaehle S, Manzoni S, van Oijen M, Wright IJ, Ciais P, van Bodegom PM, Peñuelas J, Hofhansl F, Terrer C, Soudzilovskaia NA, Midgley G, Prentice IC (2020) Organizing principles for vegetation dynamics. Nature Plants, 6, 444-453.

Franklin O, Näsholm T, Högberg P, Högberg MN (2014) Forests trapped in nitrogen limitation-An ecological market perspective on ectomycorrhizal symbiosis. New Phytologist, 203, 657-666.

Fukami T, Nakajima M (2011) Community assembly: Alternative stable states or alternative transient states? Ecology Letters, 14, 973-984.

Gentry AH (1988) Changes in plant community diversity and floristic composition on environmental and geographical gradients. Annals of the Missouri Botanical Garden, 75, 1.

Goh BS (1979) Stability in models of mutualism. The American Naturalist, 113, 261-275.

Hedin LO, Brookshire ENJ, Menge DNL, Barron AR (2009) The nitrogen paradox in tropical forest ecosystems. Annual Review of Ecology, Evolution, and Systematics, 40, 613-635.

Hilbe C, Nowak MA, Sigmund K (2013) Evolution of extortion in iterated prisoner's dilemma games. Proceedings of the National Academy of Sciences, USA, 110, 6913-6918.

Jenny H (1950) Causes of the high nitrogen and organic matter content of certain tropical forest soils. Soil Science, 69, $63-70$. 
Jiang J, Moore JAM, Priyadarshi A, Classen AT (2017) Plant-mycorrhizal interactions mediate plant community coexistence by altering resource demand. Ecology, 98, 187-197.

Koven CD, Knox RG, Fisher RA, Chambers JQ, Christoffersen BO., Davies SJ, Detto M, Dietze MC, Faybishenko B, Holm J, Huang MY, Kovenock M, Kueppers LM, Lemieux G, Massoud E, McDowell NG, Muller-Landau HC, Needham JF, Norby RJ, Powell T, Rogers A, Serbin SP, Shuman JK, Swann ALS, Varadharajan C, Walker AP, Wright SJ, Xu CG (2020) Benchmarking and parameter sensitivity of physiological and vegetation dynamics using the Functionally Assembled Terrestrial Ecosystem Simulator (FATES) at Barro Colorado Island, Panama. Biogeosciences, 17, 3017-3044.

Lin GG, McCormack ML, Ma CG, Guo DL (2017) Similar below-ground carbon cycling dynamics but contrasting modes of nitrogen cycling between arbuscular mycorrhizal and ectomycorrhizal forests. New Phytologist, 213, 1440-1451.

Lotka AJ (1925) Elements of Physical Biology. Williams \& Wilkins Company, Baltimore.

Lu MZ, Hedin LO (2019) Global plant-symbiont organization and emergence of biogeochemical cycles resolved by evolution-based trait modelling. Nature Ecology \& Evolution, 3, 239-250.

Luo YQ, Su B, Currie WS, Dukes JS, Finzi A, Hartwig U, Hungate B, McMurtrie RE, Oren R, Parton WJ, Pataki DE, Shaw MR, Zak DR, Field CB (2004) Progressive nitrogen limitation of ecosystem responses to rising atmospheric carbon dioxide. BioScience, 54, 731.

Ma ZQ, Guo DL, Xu XL, Lu MZ, Bardgett RD, Eissenstat DM, McCormack ML, Hedin LO (2018) Evolutionary history resolves global organization of root functional traits. Nature, 555, 94-97.

Martin F, Kohler A, Murat C, Veneault-Fourrey C, Hibbett DS (2016) Unearthing the roots of ectomycorrhizal symbioses. Nature Reviews Microbiology, 14, 760-773.

May RM (1974) Stability and Complexity in Model Ecosystems. Princeton University Press, Princeton.

Menge DNL, Levin SA, Hedin LO (2008) Evolutionary tradeoffs can select against nitrogen fixation and thereby maintain nitrogen limitation. Proceedings of the National Academy of Sciences, USA, 105, 1573-1578.

Menge DNL, Pacala SW, Hedin LO (2009) Emergence and maintenance of nutrient limitation over multiple timescales in terrestrial ecosystems. The American Naturalist, 173, 164-175.

Menge DNL, Wolf AA, Funk JL (2015) Diversity of nitrogen fixation strategies in Mediterranean legumes. Nature Plants, $1,15064$.

Meyer A, Grote R, Polle A, Butterbach-Bahl K (2010) Simulating mycorrhiza contribution to forest C- and N
cycling-The MYCOFON model. Plant and Soil, 327, 493-517.

Moeller HV, Neubert MG (2016) Multiple friends with benefits: An optimal mutualist management strategy? The American Naturalist, 187, E1-E12.

Montesinos-Navarro A, Segarra-Moragues JG, Valiente-Banuet A, Verdú M (2012) The network structure of plant-arbuscular mycorrhizal fungi. New Phytologist, 194, 536-547.

Neuhauser C, Fargione JE (2004) A mutualism-parasitism continuum model and its application to plant-mycorrhizae interactions. Ecological Modelling, 177, 337-352.

Noë R, Kiers ET (2018) Mycorrhizal markets, firms, and co-ops. Trends in Ecology \& Evolution, 33, 777-789.

Noë R (1990) A veto game played by baboons: A challenge to the use of the Prisoner's Dilemma as a paradigm for reciprocity and cooperation. Animal Behaviour, 39, 78-90.

Noë R, Hammerstein P (1994) Biological markets: Supply and demand determine the effect of partner choice in cooperation, mutualism and mating. Behavioral Ecology and Sociobiology, 35, 1-11.

Orwin KH, Kirschbaum MUF, St John MG, Dickie IA (2011) Organic nutrient uptake by mycorrhizal fungi enhances ecosystem carbon storage: A model-based assessment. Ecology Letters, 14, 493-502.

Peh KSH, Lewis SL, Lloyd J (2011) Mechanisms of monodominance in diverse tropical tree-dominated systems. Journal of Ecology, 99, 891-898.

Read DJ (1991) Mycorrhizas in ecosystems. Experientia, 47, 376-391.

Read DJ, Perez-Moreno J (2003) Mycorrhizas and nutrient cycling in ecosystems-A journey towards relevance? New Phytologist, 157, 475-492.

Santos FC, Pacheco JM (2005) Scale-free networks provide a unifying framework for the emergence of cooperation. Physical Review Letters, 95, 098104.

Schoener TW (1976) Alternatives to Lotka-Volterra competition: Models of intermediate complexity. Theoretical Population Biology, 10, 309-333.

Simms EL, Taylor DL (2002) Partner choice in nitrogen-fixation mutualisms of legumes and rhizobia. Integrative and Comparative Biology, 42, 369-380.

Strullu-Derrien C, Kenrick P, Pressel S, Duckett JG, Rioult JP, Strullu DG (2014) Fungal associations in Horneophyton ligneri from the Rhynie Chert (c. 407 million year old) closely resemble those in extant lower land plants: Novel insights into ancestral plant-fungus symbioses. New Phytologist, 203, 964-979.

Sulman BN, Shevliakova E, Brzostek ER, Kivlin SN, Malyshev S, Menge DNL, Zhang X (2019) Diverse mycorrhizal associations enhance terrestrial $\mathrm{C}$ storage in a global model. Global Biogeochemical Cycles, 33, 501-523.

Taylor BN, Chazdon RL, Menge DNL (2019) Successional dynamics of nitrogen fixation and forest growth in 
regenerating Costa Rican rainforests. Ecology, 100, e02637.

Tedersoo L, Bahram M, Zobel M (2020) How mycorrhizal associations drive plant population and community biology. Science, 367, eaba1223.

Terrer C, Vicca S, Hungate BA, Phillips RP, Prentice IC (2016) Mycorrhizal association as a primary control of the $\mathrm{CO}_{2}$ fertilization effect. Science, 353, 72-74.

Torti SD, Coley PD, Kursar TA (2001) Causes and consequences of monodominance in tropical lowland forests. The American Naturalist, 157, 141.

Trivers RL (1971) The Evolution of Reciprocal Altruism. The Quarterly Review of Biology, 46, 35-57.

Vitousek PM (2004) Nutrient Cycling and Limitation. Princeton University Press, Princeton.

Vitousek PM, Cassman K, Cleveland C, Crews T, Field CB, Grimm NB, Howarth RW, Marino R, Martinelli L, Rastetter EB, Sprent JI (2002) Towards an ecological understanding of biological nitrogen fixation. Biogeochemistry, 57/58, 1-45.

Vitousek PM, Field CB (1999) Ecosystem constraints to symbiotic nitrogen fixers: A simple model and its implications. Biogeochemistry, 46, 179-202.

Weng ES, Farrior CE, Dybzinski R, Pacala SW (2017) Predicting vegetation type through physiological and environmental interactions with leaf traits: Evergreen and deciduous forests in an earth system modeling framework. Global Change Biology, 23, 2482-2498.

Weng ES, Dybzinski R, Farrior CE, Pacala SW (2019) Competition alters predicted forest carbon cycle responses to nitrogen availability and elevated $\mathrm{CO}_{2}$ : Simulations using an explicitly competitive, game-theoretic vegetation demographic model. Biogeosciences, 16, 4577-4599.

Werner GDA, Cornwell WK, Cornelissen JHC, Kiers ET (2015) Evolutionary signals of symbiotic persistence in the legume-rhizobia mutualism. Proceedings of the National Academy of Sciences, USA, 112, 10262-10269.

Willis K, McElwain J (2014) The Evolution of Plants. Oxford University Press, Oxford.

Wilson DS (1975) A theory of group selection. Proceedings of the National Academy of Sciences, USA, 72, 143-146.

Wilson DS, Sober E (1994) Reintroducing group selection to the human behavioral sciences. Behavioral and Brain Sciences, 17, 585-608.

Wilson JB, Agnew ADQ (1992) Positive-feedback switches in plant communities. Advances in Ecological Research, 23, 263-336.

Wipf D, Krajinski F, Tuinen D, Recorbet G, Courty PE (2019) Trading on the arbuscular mycorrhiza market: From arbuscules to common mycorrhizal networks. New Phytologist, 223, 1127-1142.

Zheng MH, Chen H, Li DJ, Luo YQ, Mo JM (2020) Substrate stoichiometry determines nitrogen fixation throughout succession in southern Chinese forests. Ecology Letters, 23, 336-347. 